\title{
Head direction is coded more strongly than movement direction in a population of entorhinal neurons
}

Florian Raudies $^{\mathrm{a}, \mathrm{b}}$, Mark P. Brandon ${ }^{\mathrm{c}}$, G. William Chapman ${ }^{\mathrm{d}}$, and Michael E. Hasselmo ${ }^{\mathrm{a}, \mathrm{b}, \mathrm{d}}$

${ }^{a}$ Center for Computational Neuroscience and Neural Technology and ${ }^{\mathrm{b}}$ Center of Excellence for Learning in Education, Science, and Technology, Boston University, 677 Beacon Street, Boston MA 02115 USA, ${ }^{c}$ Current address: Division of Biological Science, University of California, San Diego, 9500 Gilman Drive, La Jolla, CA 92093-0357, ${ }^{\mathrm{d}}$ Department of Psychological and Brain Sciences, Center for Systems Neuroscience and Graduate Program for Neuroscience, Boston University, 2 Cummington Street, Boston, MA 02215

Abbreviated title: Coding of movement direction

Keywords: Entorhinal cortex, movement direction, grid cells, velocity controlled oscillator model, attractor model.

\section{Corresponding author:}

Florian Raudies

Center for Computational Neuroscience and Neural Technology, 677 Beacon Street Boston, MA 02215, Phone: 617-435-7918; Email: fraudies@bu.edu

Acknowledgements: MEH and FR are supported in part by the Office of Naval Research (ONR MURI N00014-10-1-0936) and by CELEST, a NSF Science of Learning Center (NSF SMA0835976). FR acknowledges support of the Office of Naval Research under the grant no ONR N00014-11-1-0535. MEH is supported in part by NIMH R01 MH60013 and MH61492. GWC and MEH acknowledge support of the Office of Naval Research under grant no ONR N0001409-1-0641. Thanks to the Moser Laboratory for providing their recorded trajectories and firing data. 
Abstract. The spatial firing pattern of entorhinal grid cells may be important for navigation. Many different computational models of grid cell firing use path integration based on movement direction and the associated movement speed to drive grid cells. However, the response of neurons to movement direction has rarely been tested, in contrast to multiple studies showing responses of neurons to head direction. Here, we analyzed the difference between head direction and movement direction during rat movement and analyzed cells recorded from entorhinal cortex for their tuning to movement direction. During foraging behavior, movement direction differs significantly from head direction. The analysis of neuron responses shows that only 5 out of 758 medial entorhinal cells show significant coding for both movement direction and head direction when evaluating periods of rat behavior with speeds above $10 \mathrm{~cm} / \mathrm{sec}$ and $\pm 30^{\circ}$ angular difference between movement and head direction. None of the cells coded movement direction alone. In contrast, 21 cells in this population coded only head direction during behavioral epochs with these constraints, indicating much stronger coding of head direction in this population. This suggests that the movement direction signal required by most grid cell models may arise from other brain structures than the medial entorhinal cortex.

\section{What makes grid cells fire?}

Grid cells in the medial entorhinal cortex fire as an animal visits an array of locations during foraging in a two-dimensional open field (Fyhn et al., 2004; Hafting et al., 2005; Barry et al., 2006; Moser and Moser, 2008). The spatial firing fields where the neuron generates action potentials tessellate the environment in a hexagonal array. The regular pattern of grid cell firing has inspired a wide range of mechanistic models of grid cell firing, primarily focused on path integration (Fuhs and Touretzky, 2006; McNaughton et al., 2006; Guanella et al., 2007; Blair et al., 2008; Burgess, 2008; Hasselmo, 2008; Burak and Fiete, 2009; Bonnevie et al., 2013; Couey et al., 2013; Pastoll et al., 2013). These models integrate the running velocity of the animal to generate the observed regular firing pattern. The integration of velocity is a critical property of both of two major classes of grid cell models, the models using velocity controlled oscillators (Burgess et al., 2007; Blair et al., 2008; Burgess, 2008; Hasselmo, 2008; Bush and Burgess, 2014) as well as models using velocity perturbations of attractor dynamics (Fuhs and Touretzky, 2006; McNaughton et al., 2006; Guanella et al., 2007; Burak and Fiete, 2009; Bonnevie et al., 
2013; Couey et al., 2013; Pastoll et al., 2013). These models have received extensive attention because they account for many features of the existing experimental data on grid cells, including theta phase precession and the intrinsic spiking frequency in autocorrelograms (Burgess, 2008) and the shared orientation and spacing of the firing fields of neighboring grid cells (Hafting et al., 2005; McNaughton et al., 2006; Moser and Moser, 2008; Burak and Fiete, 2009).

The velocity of an animal has two components, movement direction (MD) and movement speed. Many grid cell models assume that these components are available as input to the medial entorhinal cortex. For the speed component, models are supported by data from cells that change their firing rate with running speed (McNaughton et al., 1983; O'Keefe et al., 1998; Maurer et al., 2005; Wills et al., 2012). For the MD component, many articles assume that MD input is available for path integration as presented in explicit simulations (Samsonovich and McNaughton, 1995; McNaughton et al., 2006; Fuhs and Touretzky, 2006; Burgess et al., 2007; Burak and Fiete, 2009; Bonnevie et al., 2013) or in theoretical discussion (Taube et al., 1990; Sharp et al., 1995; Taube and Muller, 1998; Sargolini et al., 2006; Brandon et al., 2011). However, data on cells coding only MD and not head direction (HD) in entorhinal cortex has not been published to our knowledge. Neurons responding to allocentric HD have been described extensively in the dorsal presubiculum (Taube et al., 1990; Sharp et al., 1995; Taube, 2007), the anterior thalamus (Taube, 1995; Taube, 2007) the lateral mammillary nucleus (Stackman and Taube, 1998; Blair et al., 1998) and the medial entorhinal cortex (Sargolini et al., 2006; Brandon et al., 2011). Here, we analyze behavioral data and cell recordings to test whether MD matches HD during rat movement in an open field. In addition, we test whether any cells code for only MD without coding for HD particularly when MD and HD differ. We also test whether grid cell models function when MD is replaced by HD. 


\section{Materials and Methods}

Preparation. A detailed description of the experimental protocols used to record neuronal activity and track behavioral trajectories for data from the Hasselmo laboratory are presented in (Brandon et al., 2011) and trajectories from the Moser laboratory are presented in (Sargolini et al., 2006). Trajectories from the Moser laboratory were sampled at $50 \mathrm{~Hz}$ while the rats forage in a square box with dimensions of $1 \mathrm{~m} \times 1 \mathrm{~m}$. Trajectories from the Hasselmo laboratory were sampled at $30 \mathrm{~Hz}$ while the rat is foraging in a rectangular box with dimensions $1.13 \mathrm{~m} \times 1.28 \mathrm{~m}$. In total, 758 single-unit recordings were analyzed for this paper; 453 are from the Moser laboratory and 305 from the Hasselmo laboratory.

\section{Methods}

Surgery. Unit recording data presented here from the Hasselmo lab was obtained from six male Long-Evans rats (500-650g, 3-6 months old) that were implanted with recording drives aimed at the medial entorhinal cortex. Techniques for recording are described in detail in previous publications from the laboratory (Brandon et al., 2011; Brandon et al., 2013), but are summarized here. Prior to surgery, rats were trained to forage in open field with dimensions described above with a white cue card on one wall. For surgery, animals were anesthetized with a combination of Isoflurane and a Ketamine cocktail (Ketamine 12.92mg/ml, Acepromazine $0.1 \mathrm{mg} / \mathrm{ml}$, Xylazine $1.31 \mathrm{mg} / \mathrm{ml}$ ) and a chronic implant was affixed to the skull at approximately AP $-8.0 \mathrm{~mm}$, ML $-4.6 \mathrm{~mm}$ from bregma with 12-16 recording tetrodes (four 12.7 micron nichrome wires twisted together) aimed $\sim 25$ degrees posteriorly towards the dorsomedial entorhinal cortex. All surgical procedures followed National Institute of Health guidelines and the protocol approved by the Boston University Institutional Animal Care and Use Committee.

Unit recordings. Neurons were recorded as rats foraged in an open field environment. Data was monitored to detect grid cells, conjunctive grid by HD cells, and HD cells. In recordings from the Hasselmo lab, spiking activity was pre-amplified by unity-gain operational amplifiers on the head stage (Neuralynx, Bozeman, MT) of the animal and then amplified (5,000-20,000X) and band-pass filtered (0.3-6 kHz; Neuralynx). For signals that crossed threshold all four channels of the tetrode were digitized at $32 \mathrm{kHz}$ and recorded. Methods for cluster cutting and histological 
analysis were the same as described previously (Brandon et al., 2011). Histological analysis showed that all tetrodes passed through the medial entorhinal cortex or parasubiculum and previous work on HD cells in these structures suggests that the cells analyzed here were recorded in layers III and V.

Processing of recorded rat trajectories. In the experimental data, the trajectories of rats were tracked using two head-mounted LEDs. A video camera with $30 \mathrm{~Hz}$ sampling rate was mounted on the ceiling to track the position of one red and one green LED. Both LEDs were mounted onto the recording head stage. Rat position was defined as the centroid of the two LEDs, and rat HD was defined based on the angle between the line along the LEDs and a reference angle defined by camera orientation. For trajectories from both the Moser and Hasselmo laboratories only data from non-occluded segments were analyzed (that is, segments when the tracking LEDs were not obscured). Recording sessions from the Moser laboratory are $10 \mathrm{~min}$ or $20 \mathrm{~min}$ long and have 30,000 or 60,000 samples. Recording sessions from the Hasselmo laboratory are more variable and range from $10 \mathrm{~min}$ to $30 \mathrm{~min}$ or 18,000 to 54,000 samples. Trajectory data from the Hasselmo laboratory was recorded with Neuralynx software, which provides a position and head direction signal. Subsequently, the position and head direction was smoothed applying a Kalman filter (Brandon et al., 2013). Data from the Moser laboratory provided two tracks both smoothed with a moving mean filter to remove tracking jitter (Sargolini et al., 2006).

For each sample we computed the MD as follows. The tracked positions from the two head-mounted LEDs are denoted by $\left(x_{i}^{(1)}, y_{i}^{(1)}\right)$ and $\left(x_{i}^{(2)}, y_{i}^{(2)}\right)$ for $\mathrm{i}=1 \ldots m$, with $m$ denoting the number of samples, e.g. 20,000. Based on the jitter and angular difference distribution in the trajectory, we assumed that $\left(x_{i}^{(1)}, y_{i}^{(1)}\right)$ indicated the caudal LED (LED 1) closer to the body motion, and thus, we take the trajectory of that LED to compute the rats velocity vector as

$$
\vec{v}_{2 D}(i)=\frac{1}{\Delta t}\left(\begin{array}{c}
x_{i+1}^{(1)}-x_{i}^{(1)} \\
y_{i+1}^{(1)}-y_{i}^{(1)}
\end{array}\right),
$$

where time corresponds to the index $i$ and $\Delta t=0.02 \mathrm{sec}$ for the $50 \mathrm{~Hz}$ sampling rate or $\Delta t=0.0333 \mathrm{sec}$ for the $30 \mathrm{~Hz}$ sampling rate. The movement direction was calculated by the angle

$$
\psi(i)=\operatorname{atan} 2\left(y_{i+1}^{(1)}-y_{i}^{(1)}, x_{i+1}^{(1)}-x_{i}^{(1)}\right) .
$$


The atan 2 function returns angles in the interval of $-180^{\circ}$ to $+180^{\circ}$ and, thus, computes the arctangent for a full period.

For the data from the Hasselmo laboratory the Neuralynx recording software provides the detected head direction $\phi$ (i) per sample i. For the data from the Moser laboratory the HD was computed based on the two LED tracks as

$$
\phi(i)=\operatorname{atan} 2\left(y_{i}^{(2)}-y_{i}^{(1)}, x_{i}^{(2)}-x_{i}^{(1)}\right) \text { for } i \geq 1
$$

We computed the $0^{\circ}$ to $360^{\circ}$ angular difference between MD and $\mathrm{HD}$ as

$$
\vartheta(i)=[\psi(i)-\phi(i)]_{0}^{360^{\circ}} \text { for } i \geq 1,
$$

which subtracts the head direction from the movement direction and maps the resulting difference to the interval of 0 to $360^{\circ}$. The Table 1 summarizes the symbols in equations and their units.

Table 1 - about here

We applied four criteria for the evaluation of cell tuning for HD and MD. First, the speed criterion identifies periods of valid rat movement using the sampled speed values. Sample points were included if the rat's speed was between $10 \mathrm{~cm} / \mathrm{sec}$ and $100 \mathrm{~cm} / \mathrm{sec}$. Second, the angle criterion ensured that for evaluated sample points MD differed from HD. Sample points were included when the difference between MD and HD was above $\pm 30^{\circ}$, allowing an analysis specifically of those periods when MD and HD were not similar. Third, the spiking criterion excluded cells with only a few spikes. A recording session of a cell was only included if the number of spikes was at least $1 \%$ of the number where no speed or angle criterion was applied and a minimum of 1,000 spikes were present. Fourth, the pyramidal cell criterion excluded fast spiking interneurons by including a recording session only when the spiking frequency was within $2 \mathrm{~Hz}$ to $40 \mathrm{~Hz}$.

Statistical analysis. The response to HD or MD was plotted in polar tuning curves of firing rate to visualize the pattern of spiking dependent upon the animal's HD or MD. To construct the polar tuning curve, HD or MD was collected into bins of $6^{\circ}$. The number of spikes in each bin was divided by the time spent facing that direction (HD) or moving in that direction (MD) and multiplied by the sample rate of $30 \mathrm{~Hz}$ (or $50 \mathrm{~Hz}$ ). Polar tuning curves for Fig. 3 were smoothed using a Gaussian filter with a standard deviation of $4.5^{\circ}$ and $0^{\circ}$ mean because of the sparse data when applying our four criteria. Statistical analysis of the degree of HD selectivity or MD 
selectivity was quantified by the Watson $\mathrm{U}^{2}$ test statistic applied to the distribution of firing rates for different HDs or MDs for unsmoothed data. Cells were classified as HD cells when their Watson $\mathrm{U}^{2}$ score for $\mathrm{HD}$ was greater than 5 and their score for MD was smaller than 5. Similarly, cells were classified as MD cells when their Watson $\mathrm{U}^{2}$ score for MD was greater than 5 and their score for HD was smaller than 5. Cells with scores greater than 5 for both HD and MD were classified as joint direction (JD) tuned cells.

Velocity controlled oscillator (VCO) model. The VCO model builds upon the idea of interference between oscillations (Burgess et al., 2007; Burgess, 2008; Hasselmo, 2008). These oscillations are linked to the velocity vector $\vec{v}_{2 D}$ in the plane, which is projected onto a basis vector $\vec{b}_{k}$, specifying the path travelled along the direction of that basis vector. Many oscillatory interference models of grid cell firing use three basis vectors whose directions differ by integral multiples of $60^{\circ}$. Here, we use the three column vectors: $\vec{b}_{1}=\left(\cos \left(0^{\circ}\right), \sin \left(0^{\circ}\right)\right)^{T}$, $\vec{b}_{2}=\left(\cos \left(120^{\circ}\right), \sin \left(120^{\circ}\right)\right)^{T}$, and $\vec{b}_{3}=\left(\cos \left(240^{\circ}\right), \sin \left(240^{\circ}\right)\right)^{T}$. The superscript ' $\mathrm{T}$ ' denotes the transpose operator. The spiking of grid cells is generated by two mechanisms: First, the interference between a somatic baseline firing of $\omega$ and a speed and direction modulated dendritic firing $\omega+\omega \beta \times$ (speed for MD), with the parameter $\beta=0.00385 \mathrm{sec} / \mathrm{cm}$. This parameter $\beta$ can be interpreted as an inverse velocity and has been fitted to the measured subthreshold oscillations for neurons and their simulated grid cell spacing (Giocomo et al., 2007). The angular frequency $\omega$ is assumed to be in the theta-band provided by the medial septum and assumes the value of $\omega=2 \pi f$ with $f=7.38 \mathrm{~Hz}$ (Brandon et al., 2011). Constructive interference between the somatic and dendritic firing yields the beat frequency $\omega \beta$. Thus, each of the three basis vectors is associated with a band of firing orthogonal to the basis vector and with an inter-band distance of $s_{l}=1 /(\beta f)$. The second mechanism is the multiplicative combination (or logical AND) combination of three interfering oscillations with angular differences at intervals of $120^{\circ}$. This can be visualized as the overlay of three band patterns with orientation differences of $120^{\circ}$. Such an overlay gives rise to a hexagonal grid with a grid distance of $s_{g}=2 /(\sqrt{3} \beta f)$. Combining both mechanisms and a final threshold operation for the definition of spikes can be summarized as: 


$$
\operatorname{spike}(t)=\left\{\begin{array}{cc}
1 & \left(\prod_{k=1}^{3} \cos (\omega t)+\cos \left(\omega t+\varphi_{k}+\omega \beta \int_{0}^{t} \vec{v}_{2 D}^{T}(s) \vec{b}_{k} d s\right)\right)>\Theta . \\
0 & \text { otherwise }
\end{array} .\right.
$$

In Eq. (5) $\vec{v}_{2 D}^{T}(s) \vec{b}_{k}$ denotes the inner product (dot product) of a row-vector (the movement direction) with a column-vector (for each basis vector), which results in a scalar. A spike at time $t$ is generated when the multiplicative overlay of all three interfering oscillations or bands is above the threshold $\Theta$.

For a better comparison with the attractor model based on a twisted torus topology (Guanella et al., 2007), we added an arbitrary initialization of the phases $\varphi_{k}$ to the VCO model. Note that such initialization has to preserve the relative phase relationship between the three oscillations, thus, only a single phase value can be arbitrarily chosen, whereas the other two are determined by the basis system. In our implementation we chose $\psi$ arbitrarily, which defines the three initial phases $\varphi_{1}=(\cos \psi, \sin \psi) \vec{b}_{1}, \varphi_{2}=(\cos \psi, \sin \psi) \vec{b}_{2}$, and $\varphi_{3}=(\cos \psi, \sin \psi) \vec{b}_{3}$. This projection of the vector $(\cos \psi, \sin \psi)$ onto the basis system consisting of $\vec{b}_{1}, \vec{b}_{2}$, and $\vec{b}_{3}$ defines the phase offset in terms of a displacement corresponding to each basis vector.

An attractor model based on a twisted torus topology. The attractor model can simulate the regular hexagonal firing pattern of grid cells based on a twisted torus topology (Guanella et al., 2007). Without twisting of the torus topology a regular rectangular tessellation is created. The network consists of $n=n_{x} \times n_{y}$ model cells arranged on a grid and we show the simulation results for the cell at the index $n_{x} \times n_{y}-n_{y} / 2$. Here, we set $n_{x}=10$ and $n_{y}=9$, which gives a total of 90 simulated cells. Nodes $\vec{c}_{k}=\left(x_{i}, y_{j}\right)^{t}$ on the grid are defined by the position coordinates $x_{i}=(i-0.5) / n_{x}$ and $y_{j}=\sqrt{3} / 2(j-0.5) / n_{y}$ with $i=1 \ldots n_{x}$ and $j=1 \ldots n_{y}$ and $k$ is the linear index of $i$ and $j$, e.g. $k=j+i \times n_{y}$. Each node in the network is associated with an activity which is represented within the vector $\vec{a}(t)$, which has $n=90$ components. Weights between the nodes are defined in the matrix $W$ computed by

$$
w_{k l}(t)=I \exp \left(-\frac{\left\|\vec{c}_{k}-\vec{c}_{l}+\alpha R_{\gamma} \vec{v}_{2 D}(t)\right\|_{t r i}^{2}}{\sigma^{2}}\right)-T \text { with } k=1 \ldots \mathrm{N} \text { and } l=1 \ldots \mathrm{N} \text {. }
$$


The norm $\|\cdot\|_{t r i}$ implements a distance measure on the twisted torus topology and $I=0.3$ is the parameter for the peak synaptic strength, $T=0.05$ shifts the exponential weights at the tail end toward negative and, thus, these negative weights act as inhibition. The parameter $\alpha$ controls the input gain of the velocity and controls the grid spacing, which is approximately $1.02-0.48 \log _{2}(\alpha)$. To match the grid spacing of the recorded cells, we set this gain to $\alpha=2.5 \cdot 10^{-4}$. The matrix $R_{\gamma}$ is a rotation matrix, which controls the grid orientation $\gamma \in\left[0^{\circ}, 60^{\circ}\right]$ and $\sigma$ the standard deviation of the Gaussian. Activities $\vec{a}(t)$ are updated based on a two-step procedure:

$$
\begin{aligned}
& \vec{b}(t+1)=W(t) \vec{a}(t) \\
& \vec{a}(t+1)=\left[(1-\tau) \vec{b}(t+1)+\tau \vec{b}(t) / \sum_{i=1}^{n} \vec{a}_{i}(t)\right]^{+}
\end{aligned}
$$

The first Eq. (7a) computes the interaction between cells based on the synaptic weight matrix $\mathrm{W}(\mathrm{t})$ using a linear transfer function. The Eq. (7b) computes a exponentially weighted average with normalization against the sum of all activities that are kept positive by applying the halfwave rectification $[x]^{+}:=\max (0, x)$. The parameter $\tau$ weighs the history of activation with the current activation and is set to $\tau=0.8$. For the first step $t=0$, the components of the vector $\vec{a}$ are randomly initialized using a uniform distribution between 0 and $1 / \sqrt{ } N$. A spike for our selected model cell $k^{*}$ is generated if the activity is above the threshold level $\eta=0.1$. Formally, we define model spikes by:

$$
\text { spike }(t)=\left\{\begin{array}{ll}
1 & \vec{a}_{k^{*}}(t)>\eta \\
0 & \text { otherwise }
\end{array} .\right.
$$

We used this spiking mechanism to model the behavior of grid cells more closely, as opposed to using the continuous rate coded activation. Using a spike representation allowed us to directly use the same framework for the registration and evaluation of data as in the case of the VCO model or the recorded data for comparison.

Calculation of grid scores. The calculation of the grid scores follows the definition of (Langston et al., 2010). Intuitively, high grid scores are achieved for regular hexagonal patterns that have at least three nodes arranged in an equilateral triangle. Low grid scores are computed for non-regular structures or structures close to squared grids. Our implementation of the grid 
score calculation uses 31 pixels $\times 31$ pixels quantization mapped to the box dimensions. For nonsquare boxes the longer side maps to 31 pixels and the smaller to a fraction of 31 pixels. Spike counts are normalized by the respective occupancy in each of these pixels to define a spike rate map. Because of the unknown orientation of the grid, we compute the grid scores for eight different rotations of the spike rate map each with an increment of $3.75^{\circ}$ starting from $-15^{\circ}$ and take the maximum grid score. This method measures grid scores invariant with respect to grid orientation, e.g. when the grid's nodes are aligned with the horizontal axis.

\section{Results}

We analyzed the response of neurons in the medial entorhinal cortex as a function of allocentric HD or allocentric MD. The tracked head-motion is a super-position of body-motion and head-motion. By using the position signal of the LED closer to the center of the rat's head, we get fewer distortions from head-rotations and use this signal to approximate the body-motion. By allocentric MD, we refer to the angle of the vector drawn between sequential samples of the LED 1 positions. This approximates the directional component of the body movement of the animal. By allocentric HD, we refer to the compass direction of the main axis of the head measured relative to external coordinates (Fig. 1a). Note that allocentric HD does not refer to the egocentric position of the head relative to the body, nor does it refer to the angle relative to individual features in the environment.

Figure 1 - about here

Movement direction differs from head direction. Our first goal was to analyze behavioral data to test whether MD matches HD during rat movement. We used the position signal of the LED 1 mounted closest to the center of the head to compute MD of the center of the head and we used positions from both LEDs to compute allocentric HD (Figs. 1a and 1b). Note that we are not testing body direction, as our measure of movement direction depends upon sequential measures of LED position rather than the orientation of the body (body position or orientation is not measured in most published studies of place cells, head direction cells and grid cells). We computed the angle of the MD and HD with respect to an allocentric reference frame using 
recorded rat trajectories (Sargolini et al., 2006; Brandon et al., 2011). The HD angle and MD angles do not stay close consistently, but instead display variable differences over time (Figs. 1c and $1 \mathrm{~d})$. The rat frequently moves its head to both sides as it forages.

Cell firing rarely codes movement direction. We analyzed the firing of 758 neurons from the medial entorhinal cortex and parasubiculum (Sargolini et al., 2006; Brandon et al., 2011) for their tuning based on MD and HD by using statistical measures of significant tuning in the form of Watson $\mathrm{U}^{2}$ measures (Sargolini et al., 2006). We found that neurons were substantially better tuned for HD than for MD when applying the speed, angle, spiking, and pyramidal cell criterion (Fig. 2). Applying all four criteria we classified 21 cells as $\mathrm{HD}$ tuned $\left(\mathrm{U}^{2}(\mathrm{HD})>5\right.$ and $\left.\mathrm{U}^{2}(\mathrm{MD})<5\right)$ and 5 cells as JD tuned $\left(\mathrm{U}^{2}(\mathrm{HD})>5\right.$ and $\left.\mathrm{U}^{2}(\mathrm{MD})>5\right)$. None of the cells were clearly tuned only for MD. Based on this data we conclude that HD tuning is most common in entorhinal cortex and some cells that share both MD \& HD tuning exist. Cells tuned for only MD appear to be uncommon as we did not find a single cell with such tuning to only MD.

Figure 2 - about here

We identified stereotypical examples of differently tuned cells and show their circular histogram plots (Fig. 3). For cells from the Hasselmo laboratory we show four cells. The first exemplar cell is joint direction (JD) tuned coding both $\mathrm{HD}\left(\mathrm{U}^{2}=11.6\right)$ and $\mathrm{MD}\left(\mathrm{U}^{2}=8.4\right)$. The tuning for HD is toward north (Fig. 3a) and the tuning for MD is toward north-west (Fig. 3b). The remaining exemplar cells are HD tuned $\left(\mathrm{U}^{2}=25.4, \mathrm{U}^{2}=12.9, \mathrm{U}^{2}=24.5\right)$ coding south-west (Fig. 3c), west (Fig. 3e), and east (Fig. 3g), respectively. Their MD tuning is not statistically significant $\left(\mathrm{U}^{2}=0.9, \mathrm{U}^{2}=1.5, \mathrm{U}^{2}=1.7\right)$ (Fig. 3d, 3f, and 3h).

We selected six exemplar cells comparable in their behavior from the Moser laboratory (Fig. $3 \mathrm{i}$ - 3t). The first JD cell is tuned for both $\mathrm{HD}\left(\mathrm{U}^{2}=53.2\right)$ and $\mathrm{MD}\left(\mathrm{U}^{2}=21.5\right)$ with the HD coding south (Fig. 3i) and the MD coding east (Fig. 3j). The second example cell is tuned for both HD ( $\left.\mathrm{U}^{2}=62.6\right)$ and $\mathrm{MD}\left(\mathrm{U}^{2}=18.8\right)$ with the HD coding south-east (Fig. 3k) and the MD coding east (Fig. 31). The third exemplar cell is tuned for both $\mathrm{HD}\left(\mathrm{U}^{2}=47.9\right)$ and $\mathrm{MD}\left(\mathrm{U}^{2}=15.4\right)$ with the HD coding south-east (Fig. 3m) and the MD coding east (Fig. 3n). The fourth exemplar cell is again a JD cell tuned for $\mathrm{HD}\left(\mathrm{U}^{2}=28.1\right)$ and $\mathrm{MD}\left(\mathrm{U}^{2}=5.18\right)$ with the HD coding north (Fig. 3o) and the MD coding north-east (Fig. 3p). Notice that the tuning for MD is close to the threshold for the $U^{2}$ of 5. The last two exemplar cells are HD tuned only (Fig. 3q, 3r, 3s, 3t). 
Since HD cell tuning has been extensively studied (Taube et al., 1990; Sharp et al., 1995; Taube and Muller, 1998; Taube, 2007), we do not show additional circular histogram plots for the many other examples of cells with HD tuning.

Figure 3 - about here

Shared MD and HD may enhance response to HD. To determine the extent to which cell responses were enhanced by agreement between current $\mathrm{HD}$ and MD, we measured HD and MD tuning for subsets of the data where the measured $\mathrm{HD}$ and MD differed by variable amounts. As we focus on data where MD and HD increasingly diverge, directional tuning should weaken if there is a positive interaction between the two. We excluded angular difference values in the interval $-45^{\circ} \ldots 0^{\circ},-30^{\circ} \ldots 0^{\circ},-15^{\circ} \ldots 0^{\circ}$, none, $0^{\circ} \ldots 15^{\circ}, 0^{\circ} \ldots 30^{\circ}, 0^{\circ} \ldots 45^{\circ}$, or $-30^{\circ} \ldots+30^{\circ}$. Often cell firing appears strongest when including the angles during which MD and HD are closely aligned, as indicated by the peak in the Watsons $\mathrm{U}^{2}$ value when none of the angular differences is excluded (Fig. 4 and Fig. 5). When angular differences to the left or to the right or to both sides are excluded the Watsons $\mathrm{U}^{2}$ value drops. For HD cells this drop is stronger for the MD tuning of the cell than it is for the HD tuning (compare Fig. $4 a$ with $4 \mathrm{~b}$ and Fig. 5a with 5b), suggesting that HD cells are only mildly dependent upon MD, but MD responses are strongly dependent on HD. Because of this large drop in MD tuning and the overall smaller Watsons $\mathrm{U}^{2}$ values for $\mathrm{MD}$ tuning, these cells are classified as HD cells. Notice that for our classification we used the case with angular differences of $-30^{\circ} \ldots+30^{\circ}$ excluded. In contrast, for cells classified as JD tuned, their $\mathrm{U}^{2}$ value for the MD tuning is larger and does not drop as strongly when -30 to +30 is excluded as for HD cells (Fig. 4c and 4d and Fig. 5c and 5d). In the data set from the Moser laboratory we do not observe a drop in the $\mathrm{U}^{2}$ value of the JD cells for the MD tuning (Fig. 5d cell indices 139, 151, 159). The absence of a drop is explained by the tuning for different allocentric directions for the HD and MD. For the three JD examples the peak of the MD tuning appears in counter-clockwise orientation relative to the peak of the HD tuning (Fig. 3i-3n).

Figure 4 and 5 - about here

The velocity controlled oscillator model ( $\mathrm{VCO}$ ) and the attractor model cannot explain grid cell firing when integrating HD instead of MD. Grid cell models in both categories described here (VCO models and attractor models) have consistently assumed velocity input 
consisting of MD and movement speed (Fuhs and Touretzky, 2006; McNaughton et al., 2006; Burgess et al., 2007; Guanella et al., 2007; Blair et al., 2008; Burgess, 2008; Hasselmo, 2008; Burak and Fiete, 2009; Bonnevie et al., 2013; Couey et al., 2013; Pastoll et al., 2013). When these models use input from experimental data, the modeling input uses MD calculated from sequential positions in the data (to combine with speed), rather than the HD angle at each position in the data. Based on previous papers that cite head direction as the justification for velocity input (Fuhs and Touretzky, 2006; McNaughton et al., 2006; Burgess et al., 2007; Hasselmo et al., 2007; Burgess, 2008; Hasselmo, 2008; Burak and Fiete, 2009), it is unclear whether existing models of grid cell firing would produce grid cell firing patterns when temporally integrating HD with speed instead of MD with speed, so we tested this. As a measure for grid cell firing we use the grid score on the simulated grid cells (Langston et al., 2010). As input to the models, we replayed recorded rat trajectories to the VCO and attractor models using either the MD or the HD extracted from these trajectories combined with the movement speeds extracted from these trajectories. We provide simulation results for trajectories from the Moser laboratory (Sargolini et al., 2006). When using MD and speed as input, these two models produce grid cell firing as indicated by a high grid score. This tuning appears because the integration of velocity (MD and speed) yields spatial location relative to the starting point. We evaluate the firing patterns and grid scores for the VCO model and the attractor model for three types of simulations. In the first type of simulation (Fig. 6a and 6e), the models use the MD and speed of the rat calculated from one LED track. That is, the simulations use the velocity vector $v$ as shown in the model Eq. 5 and Eq. 6. In the second type of simulation (Fig. $6 \mathrm{~b}$ and $6 \mathrm{f}$ ), the models use HD in place of MD, where HD is calculated from the same LED tracks, that is, replacing $v$ with $\varphi$ in Eq. 5 and Eq. 6 (speed is still calculated from a single LED). In the third type of simulation (Figs. 6c, 6d, 6g, and 6h), the models use a temporally smoothed version of the HD signal. For all types of simulations, spikes were evaluated according to the spatial location of the LED tracks. Both models produce high grid scores using the MD with speed as input (Fig. 6a and 6e) and low grid scores when using either the HD with speed as input (Fig. 6b and 6f) or temporally smoothed HD with speed as input (Fig. 6c, 6d, 6g, and 6h). For smoothing we used a simple box-filter extending over a temporal window of 4 or 16 seconds. To rule out that this as an artifact of specific recorded trajectories or initialization of the models, we repeated the evaluation for six trajectories with 50 runs for three trajectories (Table 2). This illustrates that 
the difference between HD and MD is large enough that HD cannot be used as an effective proxy for MD, even when temporally smoothed. This simulation confirms that the HD input cannot replace the MD input in either the VCO model or the attractor model and that this is not an artifact of the chosen trajectory or the initialization of the model.

Figure 6 - about here

Table 2 - about here

\section{Discussion}

Only 5 out of the 758 cells recorded in medial entorhinal cortex code both movement direction (MD) and head direction (HD). No cell responds purely to MD, though a large number (21 cells) respond selectively to $\mathrm{HD}$, indicating a predominant coding of $\mathrm{HD}$. In addition, our analysis of movement behavior shows that HD cannot replace MD for path integration in models of grid cell firing. Thus, grid cell models require coding of MD in structures, e.g. beyond medial entorhinal cortex (Wedlay et al., 2011), or may require alternate means of path integration that do not involve explicit coding of MD. The MD may be coded in structures other than entorhinal cortex and still drive grid cells in medial entorhinal cortex. Note that the two data sets (Sargolini et al., 2006; Brandon et al., 2011) were recorded with different software (Axona versus Neuralynx) so that the similar results rule out features due to the tracking techniques or tracking software.

We did not find a single cell selective for MD alone (Watson $\mathrm{U}^{2}>5$ ) that was not also selective for HD (Watson $\mathrm{U}^{2}<5$ ). This analysis is consistent with previous studies reporting that cells code HD and do not respond to other variables (Taube et al., 1990; Sharp et al., 1995; Taube, 1995; Taube and Muller, 1998; Blair et al., 2008), but most prior experimental studies did not quantitatively compare HD and MD.

One previous study showed a dependence of HD cells on running speed. In that study, HD cells fire more strongly at high running speed when rats point their head in the direction that the HD cell is tuned for (Taube et al., 1990). This is somewhat similar to our finding that the response of $\mathrm{HD}$ cells is stronger when including the periods of time when MD is close in angle to HD. However, such dependency of HD cells on running speed in the previous study was independent of the MD and, thus, cannot provide MD information. 
Head direction cannot replace MD for movement behavior of rats. Movement direction and HD are clearly distinct for rat trajectories (Fig. 1). Integration over different time windows does not align the HD with the MD; in contrast, the two directions become more disparate. We also show that neither the VCO nor the attractor model produce grid cell firing when integrating $\mathrm{HD}$ in place of MD. These grid cell models have to include a mechanism to account for the MD especially when the rat is not running forward relative to head direction. Our data indicates that coding of MD is not prominent in the medial entorhinal cortex, and HD cannot directly replace MD in existing models using path integration. This does not rule out MD being coded by mechanisms other than firing rate or in other structures such as the anterior thalamus, medial septum and hippocampus (Wedlay et al., 2011). In addition, this does not rule out other methods of updating location that do not require an explicit code of MD.

Movement direction can be extracted from optic flow facilitated by HD and speed signals both coded in the firing of entorhinal cells. The temporal derivative $\mathrm{dHD} / \mathrm{dt}$ provides a rotational velocity signal in the 2D plane. Given this rotational signal, optic flow information can be easily used to extract the MD. When this MD is combined with the speed signal a 2D velocity signal in the same 2D plane that HD code for is available as input to the grid cells. Thus, HD together with running speed and optic flow can provide MD to models of grid cell firing (Raudies et al., 2012).

Path integration might happen in brain structures other than entorhinal cortex. Retrosplenial cortex is necessary for path integration (Sutherland, 1988) and if lesioned leads to impairment of the head direction cell firing in the anterodorsal thalamus (Clark et al., 2010). Thus, retrosplenial cortex is one candidate area to look for MD tuning. Rats with parietal cortex lesions had inaccurate return trips to a refuge, given only self-movement cues (Parron and Save, 2004). This indicates that parietal cortex is involved in path integration. Path integration mechanisms might also occur in the hippocampus (McNaughton et al., 1996). Thus, parietal cortex and the hippocampus are additional candidate brain regions to analyze for MD tuning.

Idiothetic cues provide HD and maybe MD. Motor efference copies and proprioceptive signals help to maintain a stable HD signal (Stackman et al., 2003). Besides these internal signals and representations, sensory perception measuring changes of the rat's movement can provide HD and MD. Angular acceleration is sensed by the semicircular canal. When temporally integrated once, head velocity can be obtained, which is coded by cells in the dorsal tegmental 
nucleus (Bassett, 2001; Sharp et al., 2001). Another sensory cue is optic flow, which can update the anterodorsal thalamus coding of HD (Yoder et al., 2011; Arleo et al., 2013). Our analysis of behavioral data shows that HD varies too much from MD to be used as a replacement for MD in path integration. However, idiothetic cues might also lead to coding of MD, aside from the coding of HD. For example, efference copies for motor commands could be used directly for path integration. The semicircular canals provide translational acceleration information, that integrated twice give an estimate of position. Optic flow enables the estimation of MD (Perrone, 1992).

Allothetic visual cues strongly influence the firing of neurons in entorhinal cortex and hippocampus, as rotations of landmark information cause rotations in the firing properties of place cells (Muller and Kubie, 1987; Knierim et al., 1998), grid cells (Hafting et al., 2005), and head direction cells (Taube et al., 1990). Similarly, enlarging or shrinking the size of the environment by moving barriers causes a compression or expansion of the firing fields of place cells (O'Keefe and Burgess, 1996) and grid cells (Barry et al., 2007). Adding barriers to the environment that require the animal to move in constrained trajectories causes grid cells and place cells to respond more strongly to the one dimensional position of the animal along a trajectory (Derdikman et al., 2009; Gupta et al., 2014).

This suggests that environmental cues such as landmarks affect the firing locations of grid cells, HD cells, and place cells. Observed landmarks, whose positions have been memorized previously, can be used for self-localization through triangulation. For a rat running on a flat plane, the visual angles toward the landmarks are sufficient to estimate the position of self within the reference of the landmarks. In summary, visual input as a cue can generate spatial responses of grid cells, though visual input is not the only available information.

Grid cells and place cells continue to fire in darkness (O'Keefe and Nadel, 1978; Hafting et al., 2005), or can be driven by self-motion cues without visual input (Kinkhabwala et al., 2011; Chen, 2012). Thus, most likely, grid cells integrate multiple sensory cues including visual and sensorimotor cues that may directly update grid cell firing based on relative cue movement without an intermediate computation of the animal's MD. Extended models of grid cell firing should include the influence of sensory cues. 


\section{References}

Arleo A, Dejean C, Allegraud P, Khamassi M, Zugaro MB, Wiener SI (2013) Optic flow stimuli update anterodorsal thalamus head direction neural activity in rats. J Neurosci 33:1679016795.

Barry C, Hayman R, Burgess N, Jeffery KJ (2007) Experience-dependent rescaling of entorhinal grids. Nat Neurosci 10:682-684.

Barry C, Lever C, Hayman R, Hartley T, Burton S, O'Keefe J, Jeffery K, Burgess N (2006) The boundary vector cell model of place cell firing and spatial memory. Rev Neurosci 17:7197.

Blair HT, Cho J, Sharp PE (1998) Role of the lateral mammillary nucleus in the rat head direction circuit: a combined single unit recording and lesion study. Neuron 21(6): 13871397.

Blair HT, Gupta K, Zhang K (2008) Conversion of a phase- to a rate-coded position signal by a three-stage model of theta cells, grid cells, and place cells. Hippocampus 18:1239-1255.

Bonnevie T, Dunn B, Fyhn M, Hafting T, Derdikman D, Kubie JL, Roudi Y, Moser EI, Moser MB (2013) Grid cells require excitatory drive from the hippocampus. Nat Neurosci 16:309-317.

Brandon MP, Bogaard AR, Schultheiss NW, Hasselmo ME (2013) Segregation of cortical head direction cell assemblies on alternating theta cycles. Nat Neurosci 16:739-748.

Brandon MP, Bogaard AR, Libby CP, Connerney MA, Gupta K, Hasselmo ME (2011) Reduction of theta rhythm dissociates grid cell spatial periodicity from directional tuning. Science 332:595-599.

Burak Y, Fiete IR (2009) Accurate path integration in continuous attractor network models of grid cells. PLoS Comput Biol 5:e1000291.

Burgess N (2008) Grid cells and theta as oscillatory interference: theory and predictions. Hippocampus 18:1157-1174.

Burgess N, Barry C, O'Keefe J (2007) An oscillatory interference model of grid cell firing. Hippocampus 17:801-812.

Bush D, Burgess N. (2014) A Hybrid Oscillatory Interference/Continuous Attractor Network Model of Grid Cell Firing. J Neurosci 34:5065-5079.

Chen J, King, J.A., Burgess, N., and O'Keefe, N. (2012) How vision and movement combine in the hippocampal place code Proc Natl Acad Sci U S A, doi:2010.1073/pnas.1215834110.

Clark, B. J., Bassett, J. P., Wang, S. S., and Taube, J. S. (2010). Impaired head direction cell representation in the anterodorsal thalamus after lesions of the retrosplenial cortex. J. Neurosci. 30, 5289-5302.

Couey JJ, Witoelar A, Zhang SJ, Zheng K, Ye J, Dunn B, Czajkowski R, Moser MB, Moser EI, Roudi Y, Witter MP (2013) Recurrent inhibitory circuitry as a mechanism for grid formation. Nat Neurosci 16:318-324.

Derdikman D, Whitlock JR, Tsao A, Fyhn M, Hafting T, Moser MB, Moser EI (2009) Fragmentation of grid cell maps in a multicompartment environment. Nat Neurosci 12:1325-1332.

Fuhs MC, Touretzky DS (2006) A spin glass model of path integration in rat medial entorhinal cortex. J Neurosci 26:4266-4276.

Fyhn M, Molden S, Witter MP, Moser EI, Moser MB (2004) Spatial representation in the entorhinal cortex. Science 305:1258-1264. 
Giocomo LM, Zilli EA, Fransen E, Hasselmo ME (2007) Temporal frequency of subthreshold oscillations scales with entorhinal grid cell field spacing. Science 315:1719-1722.

Guanella A, Kiper D, Verschure P (2007) A model of grid cells based on a twisted torus topology. Int J Neural Syst 17:231-240.

Gupta K, Beer NJ, Keller LA, Hasselmo ME (2014) Medial Entorhinal Grid Cells and Head Direction Cells Rotate with a T-Maze More Often During Less Recently Experienced Rotations. Cereb Cortex. 6:1630-44.

Hafting T, Fyhn M, Molden S, Moser MB, Moser EI (2005) Microstructure of a spatial map in the entorhinal cortex. Nature 436:801-806.

Hasselmo ME (2008) Grid cell mechanisms and function: contributions of entorhinal persistent spiking and phase resetting. Hippocampus 18:1213-1229.

Hasselmo ME, Giocomo LM, Zilli EA (2007) Grid cell firing may arise from interference of theta frequency membrane potential oscillations in single neurons. Hippocampus 17:1252-1271.

Kinkhabwala AA, Aronov D, Tank DW (2011) The role of idiothetic (self-motion) information in grid cell firing. Soc Neurosci Abstr 37:729.716.

Knierim JJ, Kudrimoti HS, McNaughton BL (1998) Interactions between idiothetic cues and external landmarks in the control of place cells and head direction cells. J Neurophysiol 80:425-446.

Langston RF, Ainge JA, Couey JJ, Canto CB, Bjerknes TL, Witter MP, Moser EI, Moser MB (2010) Development of the spatial representation system in the rat. Science 328:15761580.

Maurer AP, Vanrhoads SR, Sutherland GR, Lipa P, McNaughton BL (2005) Self-motion and the origin of differential spatial scaling along the septo-temporal axis of the hippocampus. Hippocampus 15:841-852.

McNaughton BL, Barnes CA, O'Keefe J (1983) The contributions of position, direction, and velocity to single unit-activity in the hippocampus of freely-moving rats. Experimental Brain Research 52:41-49.

McNaughton BL, Barnes CA, Gerrard JL, Gothard K, Jung MW, Knierim JJ, Kudrimoti H, Qin Y, Skaggs WE, Suster M, and Weaver KL (1996) Deciphering the hippocampal polyglot: the hippocampus as a path integration system. J Exp Biology 199:173-185.

McNaughton BL, Battaglia FP, Jensen O, Moser EI, Moser MB (2006) Path integration and the neural basis of the 'cognitive map'. Nat Rev Neurosci 7:663-678.

Moser EI, Moser MB (2008) A metric for space. Hippocampus 18:1142-1156.

Muller RU, Kubie JL (1987) The effects of changes in the environment on the spatial firing of hippocampal complex-spike cells. J Neurosci 7:1951-1968.

O'Keefe J, Nadel L (1978) The Hippocampus as a Cognitive Map. Oxford, UK: Oxford University Press.

O'Keefe J, Burgess N (1996) Geometric determinants of the place fields of hippocampal neurons. Nature 381:425-428.

O'Keefe J, Burgess N, Donnett JG, Jeffery KJ, Maguire EA (1998) Place cells, navigational accuracy, and the human hippocampus. Philos Trans R Soc Lond B Biol Sci 353:13331340.

Pastoll H, Solanka L, van Rossum MC, Nolan MF (2013) Feedback inhibition enables thetanested gamma oscillations and grid firing fields. Neuron 77:141-154. 
Perrone J (1992) Model for the computation of self-motion in biological systems. J of the Opt Soc of Am A 9:177-192.

Raudies, F., Mingolla, E., and Hasselmo, M.E. (2012) Modeling the Influence of Optic Flow on Grid Cell Firing in the Absence of Other Cues. Journal of Computational Neuroscience. 33(3), 465-493

Sargolini F, Fyhn M, Hafting T, McNaughton BL, Witter MP, Moser MB, Moser EI (2006) Conjunctive representation of position, direction, and velocity in entorhinal cortex. Science 312:758-762.

Sharp PE, Blair HT, Etkin D, Tzanetos DB (1995) Influences of vestibular and visual motion information on the spatial firing patterns of hippocampal place cells. J of Neurosci 15:173-189.

Stackman RW, Taube JS (1998) Firing properties of rat lateral mammillary single units: head direction, head pitch and angular head velocity. J. Neurosci. 18(21): 9020-9037.

Stackman RW, Golob EJ, Bassett JP, Taube JS (2003) Passive transport disrupts directional path integration by head direction cells. J. Neurophysiol. 90(5): 2862-2874.

Taube JS (1995) Head direction cells recorded in the anterior thalamic nuclei of freely moving rats. J Neurosci 15:70-86.

Taube JS (2007) The head direction signal: Origins and sensory-motor integration. Annu. Rev. Neurosci. 30: 181-2007.

Taube JS, Muller RU (1998) Comparisons of head direction cell activity in the postsubiculum and anterior thalamus of freely moving rats. Hippocampus 8:87-108.

Taube JS, Muller RU, Ranck JB, Jr. (1990) Head-direction cells recorded from the postsubiculum in freely moving rats. I. Description and quantitative analysis. J Neurosci $10: 420-435$.

Wedlay AC, Shlifer IG, Bloom ML, Zhang K, Blair HT (2011) Cosine directional tuning of theta cell burst frequencies: evidence for spatial coding by oscillatory interference. J of Neurosci 31:16157-16176.

Wills TJ, Barry C, Cacucci F (2012) The abrupt development of adult-like grid cell firing in the medial entorhinal cortex. Front Neural Circuits 6:21.

Yoder RM, Clark BJ, Brown JE, Lamia MV, Valerio S, Shinder ME, Taube JS. (2011) Both visual and idiothetic cues contribute to head direction cell stability during navigation along complex routes. J Neurophysiol. 105(6):2989-3001. 


\section{Figure Legends}

Fig 1. Schematic demonstration of the difference in definition of movement direction (MD) and head direction (HD). (a) Sketch of a rat whose HD differs from the MD. LED 1 is assumed to be caudally mounted and LED 2 is assumed to have a rostral mount. (b) An idealized trajectory with three sampling times shows the computation of HD and MD from the location of two LEDs (LED1 and LED2) detected at different time points (four time points shown for LED 1 and three time points for LED 2). (c) and (d) Angle of $\mathrm{HD}$ and MD over the period of a few seconds within the interval of $0^{\circ}$ to $720^{\circ}$. Discontinuities in the curve plot between $0^{\circ}$ and $720^{\circ}$ are depicted by circles. In circular space the values $0^{\circ}, 360^{\circ}$, and $720^{\circ}$ are equivalent.

Fig 2. A large number of cells show significant coding of head direction (HD) (square symbols), and a smaller number show significant tuning to both HD and movement direction (MD) (triangle symbols). (a) Watson $\mathrm{U}^{2}$ for the population of 305 cells from the Hasselmo laboratory and (b) Watson $\mathrm{U}^{2}$ for the population of 453 cells from the Moser laboratory. Plots show the Watson $\mathrm{U}^{2}$ for MD tuning versus Watson $\mathrm{U}^{2}$ for HD tuning of the same cell. Both axes are log-scaled as are the gray-values that represent the firing rate of each cell in $\mathrm{Hz}$. Cells whose Watson $\mathrm{U}^{2}$ pair is below the diagonal primarily encode $\mathrm{HD}$, whereas those above the diagonal primarily encode MD. The distribution of cells is predominantly biased toward statistical encoding of HD rather than MD. Numbers denote cell indices. Dashed lines mark the threshold of 5 for the $\mathrm{U}^{2} \mathrm{~s}$ that we used to categorize HD and MD cells.

Fig 3. Exemplar cells from the Hasselmo and Moser laboratories. (a-b) Circular histogram of a cell tuned for HD and MD, (c-h) three cells tuned only for HD. (i-p) Circular histograms of cells tuned for HD and MD, (q-t) two cells tuned only for HD. In all exemplar plots we applied the speed, angle, spiking, and pyramidal cell criteria. Histogram plots use a $6^{\circ}$ bin size to count spikes and occupancy and show the spike rate in $\mathrm{Hz}$ (calculated by taking the count of spikes divided by the count of occupancy multiplied by the sampling rate). For display, plots are smoothed by a Gaussian with standard deviation of $4.5^{\circ}$.

Fig. 4. The influence of periods of shared behavioral angle on cell firing is shown by analysis of the statistical tuning response (Watson U2) to HD or MD during exclusion of behavioral periods with different amounts of angular difference between HD and MD. This figure shows the effect for the data from the Hasselmo laboratory. (a) Watson $\mathrm{U}^{2}$ values for HD tuning in HD tuned cells and (b) for MD tuning in HD tuned cells. (c) Watson $\mathrm{U}^{2}$ values for HD tuning in JD tuned cells and (d) for MD tuning in JD tuned cells. Symbols indicate the cell according to the legend to the right. 
Fig. 5. Analysis of tuning properties during exclusion of behavioral periods with different amounts of angular difference for the data from the Moser laboratory. (a) Watson $\mathrm{U}^{2}$ values for HD tuning in HD tuned cells and (b) for MD tuning in HD tuned cells. (c) Watson $\mathrm{U}^{2}$ values for HD tuning in HD tuned cells and (d) for MD tuning in JD tuned cells. Symbols indicate the cell according to the legend to the right.

Fig 6. Simulations of the velocity controlled oscillator (VCO) model (top row) and attractor model (bottom row) with different types of input. (a and e): Simulations show that effective grid cell firing is obtained when using input of a rat's tracked movement direction (MD) and speed, which is the velocity vector $v$ in Eqs. 7 and 8. (b and f): In contrast, simulations do not generate grid cell firing patterns when input consists of a rat's speed combined with tracked head direction (HD) in place of MD, (replacing $v$ with $\varphi$ in Eqs. 7 and 8). (c) and (d): Simulations still do not generate grid cell firing patterns when using input of HD that has been temporally smoothed over $4 \mathrm{sec}$. (d) and (h): Temporal smoothing of HD over $16 \mathrm{sec}$ does not improve the grid cell firing patterns. 


\begin{tabular}{lll}
\hline Symbols in equations & Description & Unit \\
\hline \hline$\left(x_{i}^{(1)}, y_{i}^{(1)}\right)$ & LED closer to the body of the rat. & $\mathrm{cm}$ \\
\hline$\left(x_{i}^{(2)}, y_{i}^{(2)}\right)$ & LED further away from the body. & $\mathrm{cm}$ \\
\hline$m$ & Number of samples from a recording session. & none \\
\hline$\phi$ & Head direction. & $\circ$ \\
\hline$\psi$ & Movement direction. & $\mathrm{cm} / \mathrm{sec}$ \\
\hline$\vec{v}_{2 D}$ & Velocity in a 2D plane. & $\circ$ \\
\hline$\vartheta$ & Angle of movement to head direction. & $\mathrm{sec}$ \\
\hline$t$ & Time. &
\end{tabular}

Velocity controlled oscillator model

\begin{tabular}{lll}
\hline \hline$\vec{b}_{1}, \vec{b}_{2}, \vec{b}_{3}$ & Basis vectors. & none \\
\hline$\omega=2 \pi f$ & Angular frequency. & $\mathrm{Hz}$ \\
\hline$\beta$ & Parameter that maps velocity to grid spacing. & sec/cm \\
\hline$\varphi_{k}$ & Initial phase value. & $\circ$ \\
\hline$\Theta$ & Firing threshold. & none \\
\hline
\end{tabular}

Attractor model based on a twisted torus topology

\begin{tabular}{lll}
\hline \hline$n$ & Number of model neurons. & none \\
\hline$n_{x}$ & Model neurons in the horizontal direction in the 2D ground plane. & none \\
\hline$n_{y}$ & Model neurons in the vertical direction. & none \\
\hline$\vec{c}_{k}=\left(x_{i}, y_{j}\right)$ & Horizontal and vertical coordinate of a node. & none \\
\hline$w_{k l}$ & Weights between nodes. & none \\
\hline$I$ & Peak of synaptic strength. & none \\
\hline$T$ & Controls excitatory versus inhibitory strength for weights. & none \\
\hline$\alpha$ & Input gain which scales between environment size and node distance. & $1 / \mathrm{cm}$ \\
\hline$\gamma$ & Grid orientation. & $\circ$ \\
\hline
\end{tabular}

Table 1. Description of identifiers and their units, if applicable. 


\begin{tabular}{|l|l|l|l|l|l|l|l|l|l|l|}
\hline Rat / recording & \multicolumn{9}{|c|}{$\begin{array}{c}\text { Grid scores for velocity controlled } \\
\text { oscillator model }\end{array}$} \\
\cline { 2 - 11 } & Data & MD & $\begin{array}{l}\text { HD } \\
0 \mathrm{~s}\end{array}$ & $\begin{array}{l}\text { HD } \\
4 \mathrm{~s}\end{array}$ & $\begin{array}{l}\text { HD } \\
16 \mathrm{~s}\end{array}$ & Data & MD & HD & HD & HD \\
$0 \mathrm{~s}$ & $\mathrm{~s}$ & $16 \mathrm{~s}$ \\
\hline $\begin{array}{l}11084- \\
09030501 \mathrm{~T} 1 \mathrm{C} 2\end{array}$ & 1.46 & 1.85 & 0.38 & -0.22 & -0.73 & 1.46 & 1.80 & 0.26 & 0.04 & -0.71 \\
\hline $\begin{array}{l}11138- \\
13040502 \mathrm{~T} 5 \mathrm{C} 1\end{array}$ & 1.63 & 1.84 & 0.04 & -0.06 & -0.72 & 1.63 & 1.83 & 0.34 & 0.20 & -0.72 \\
\hline $\begin{array}{l}11265- \\
09020601 \mathrm{~T} 2 \mathrm{C} 3\end{array}$ & 1.51 & 1.75 & -0.06 & -0.06 & -0.72 & 1.51 & 1.87 & 0.29 & 0.03 & -0.72 \\
\hline
\end{tabular}

Table 2. Grid scores for the recorded and simulated grid cells using either input of movement direction (MD) combined with speed or input of head direction (HD) combined with speed. Simulations were performed with the velocity controlled oscillator (VCO) model or attractor model. Except for the values in the data columns, all numbers are mean values for 50 trials of signed grid scores. 
Figure's

Figure 1
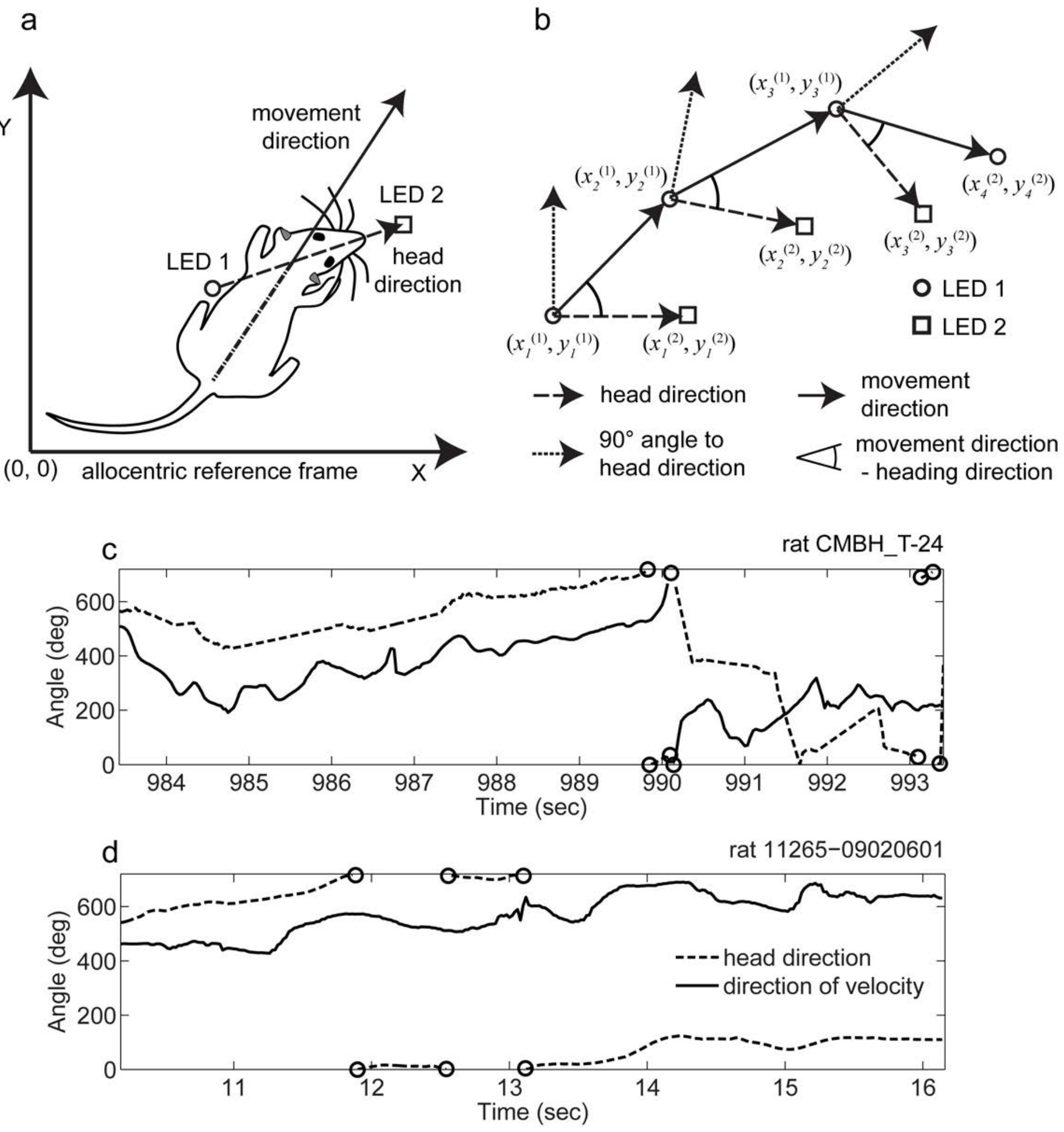
Figure 2

a Brandon et al. 2011

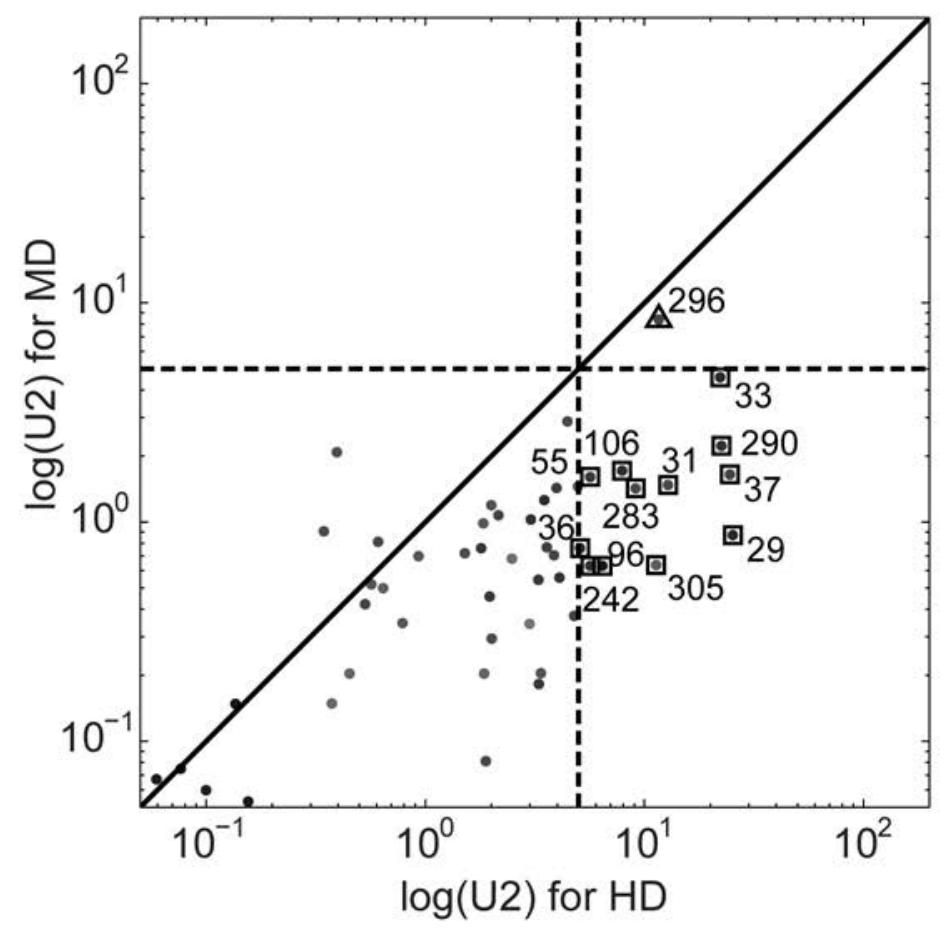

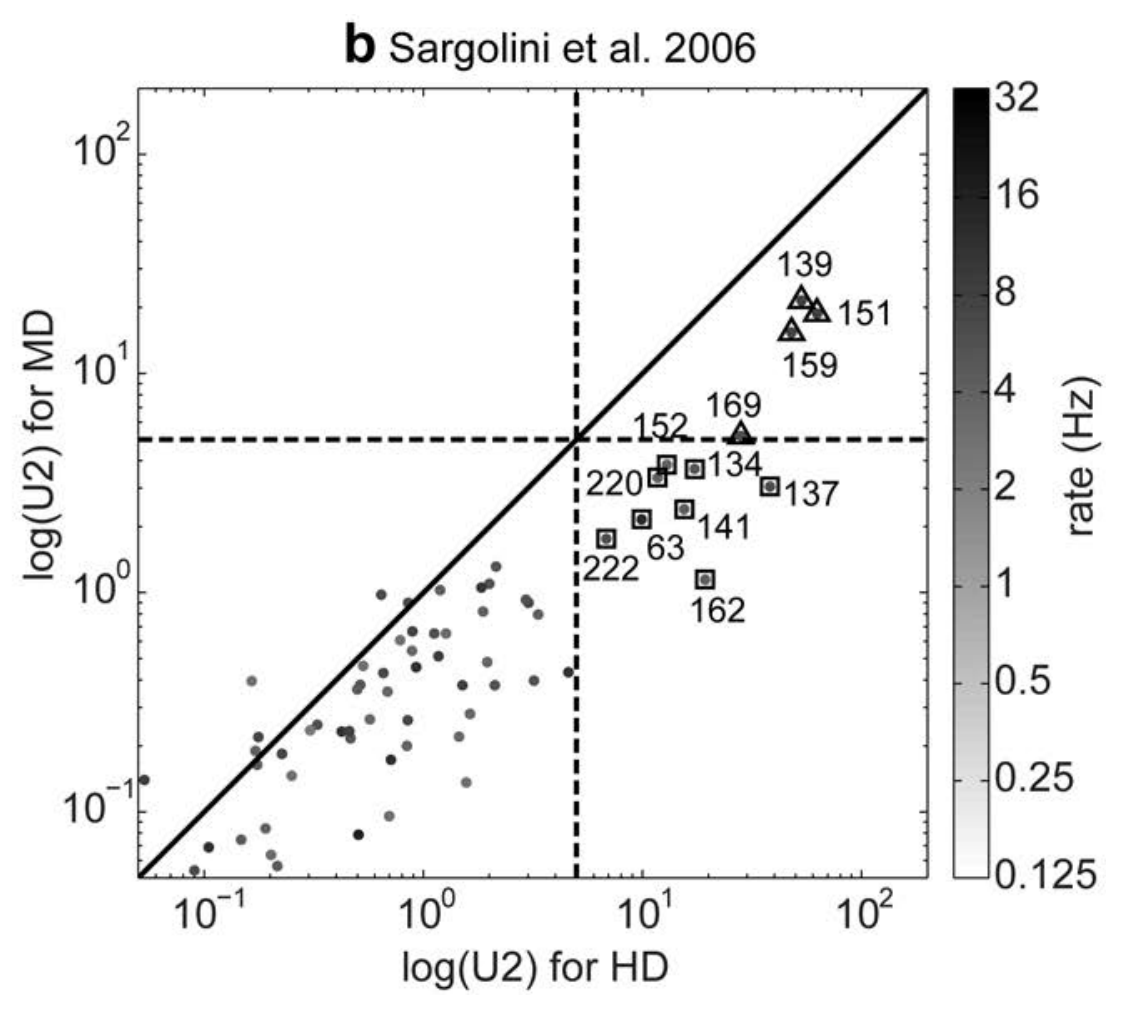


Figure 3

Brandon et al., 2011

HD tuning

MD tuning

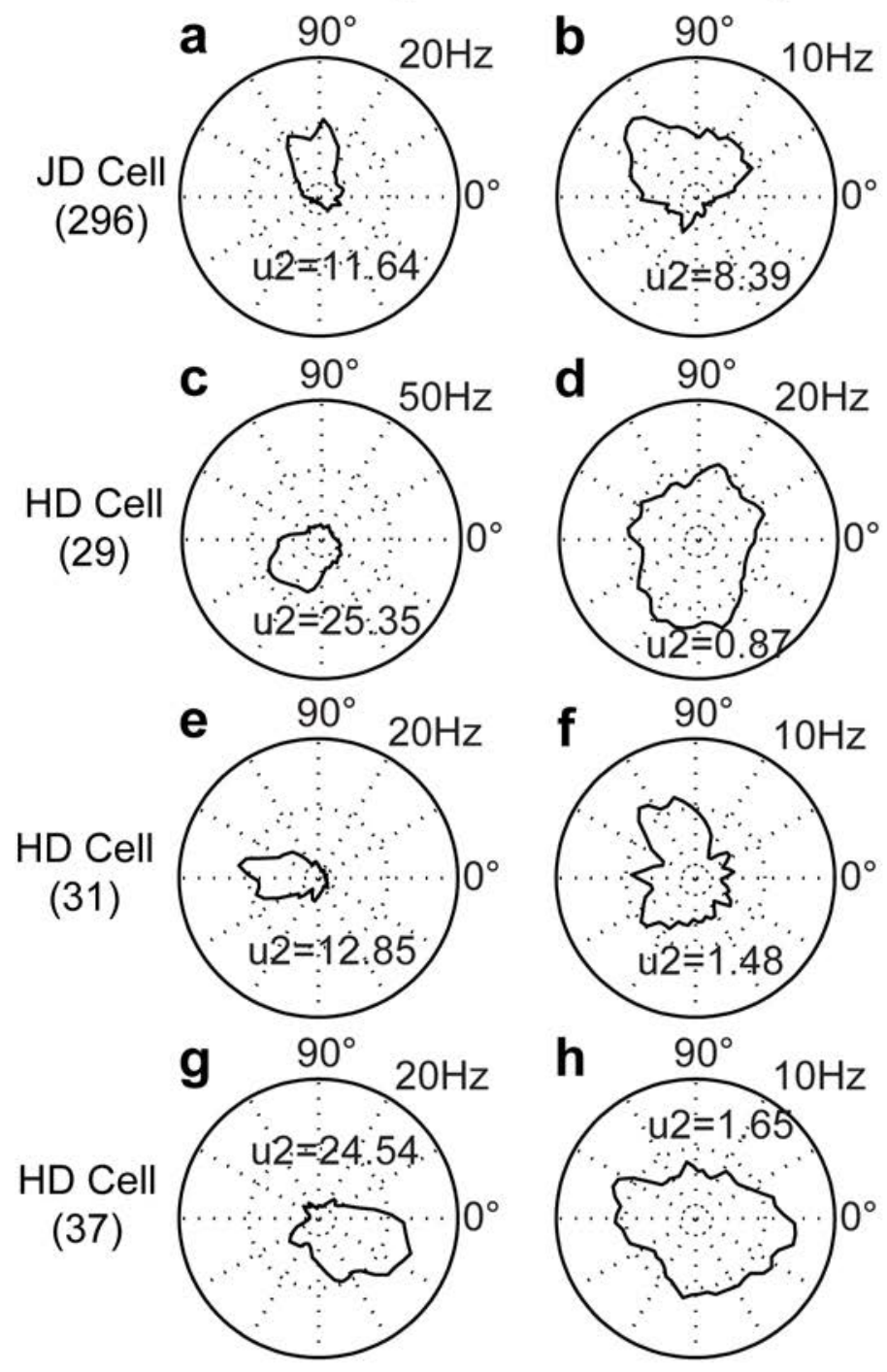

Sargolini et al. 2006 HD tuning
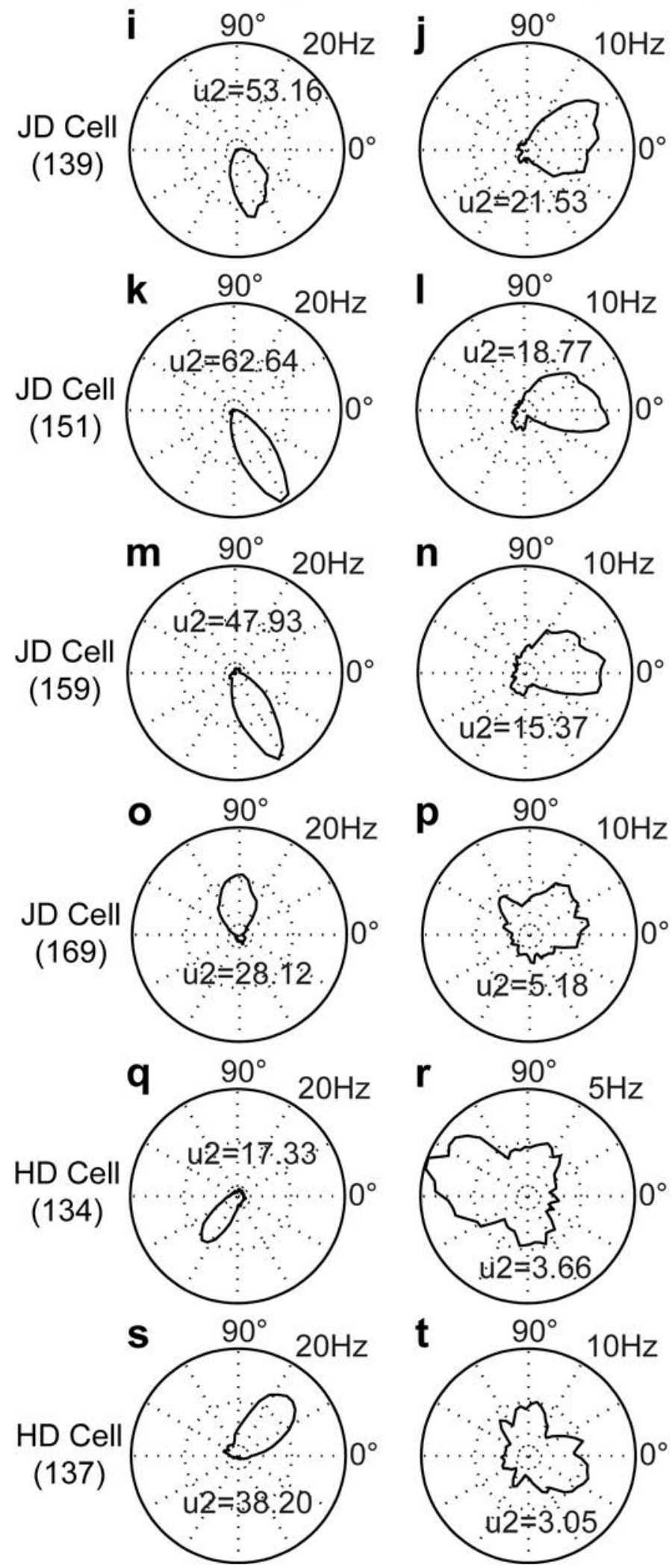
Figure 4
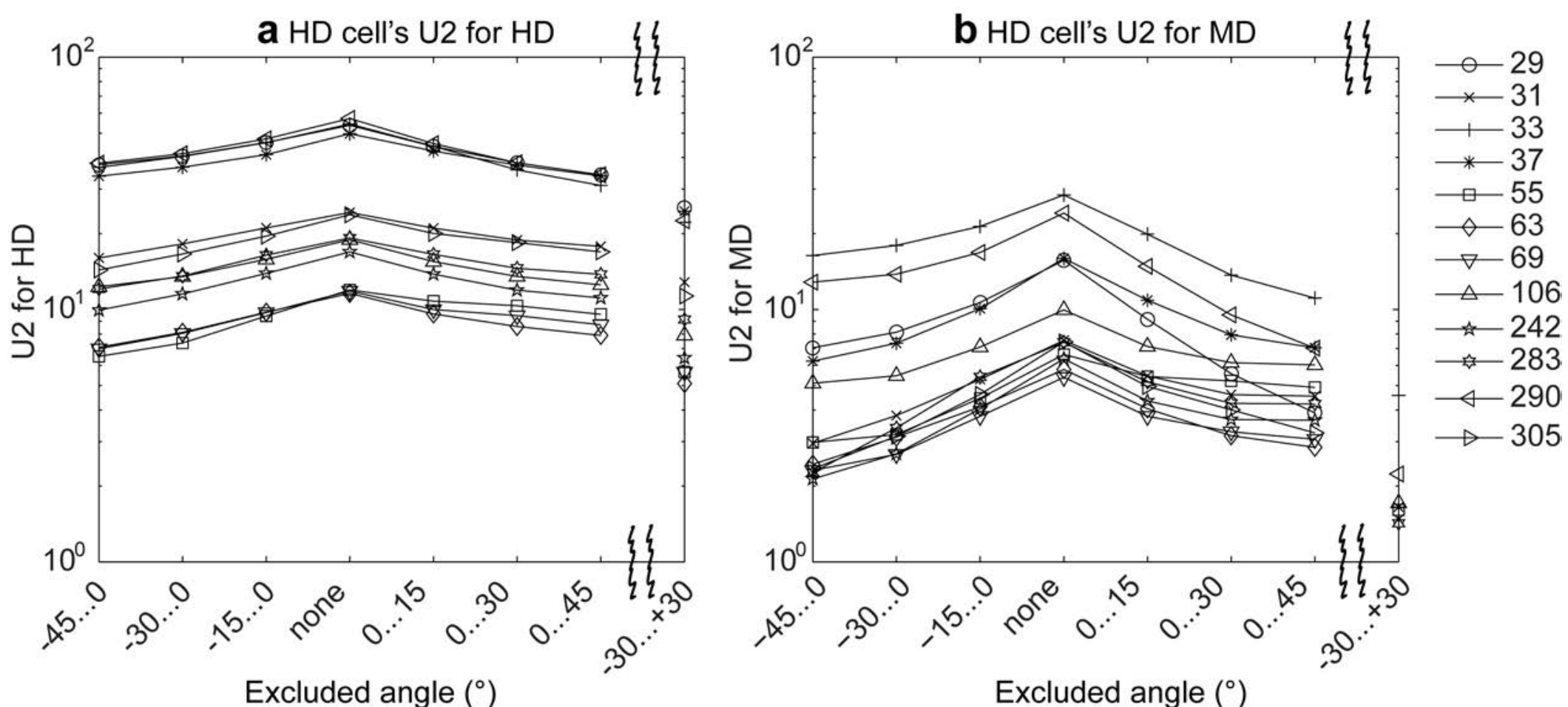

Excluded angle $\left({ }^{\circ}\right)$

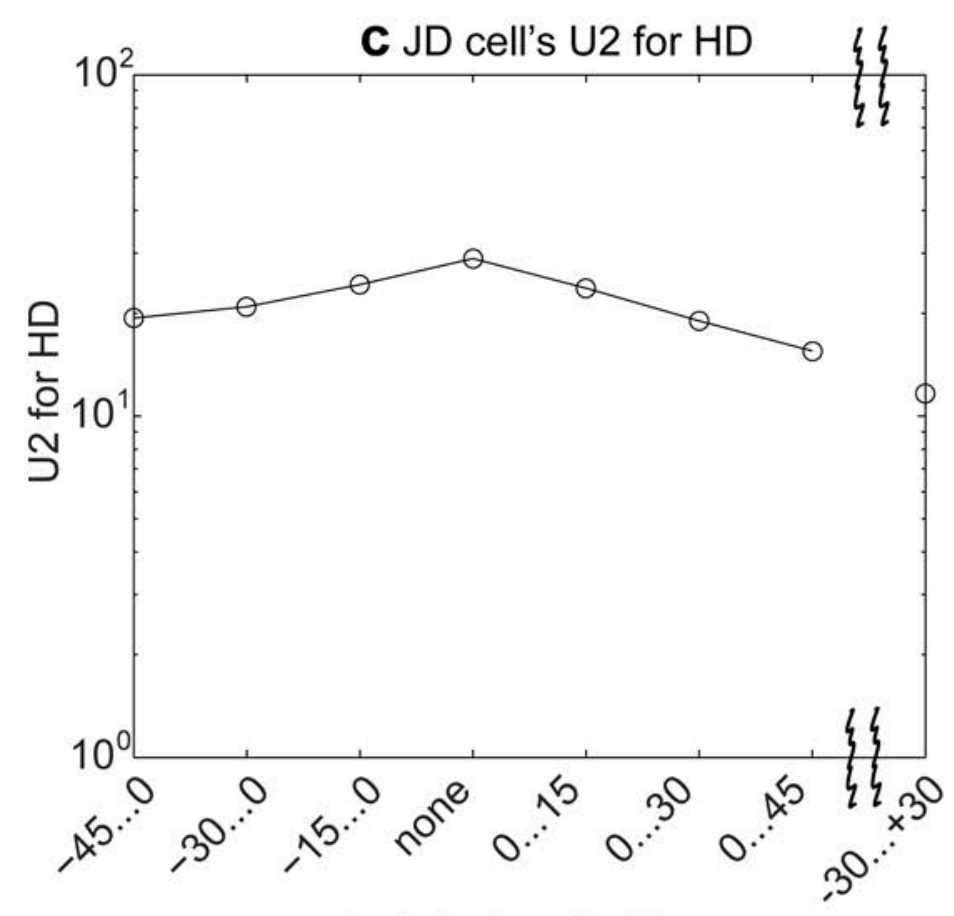

Excluded angle $\left({ }^{\circ}\right)$

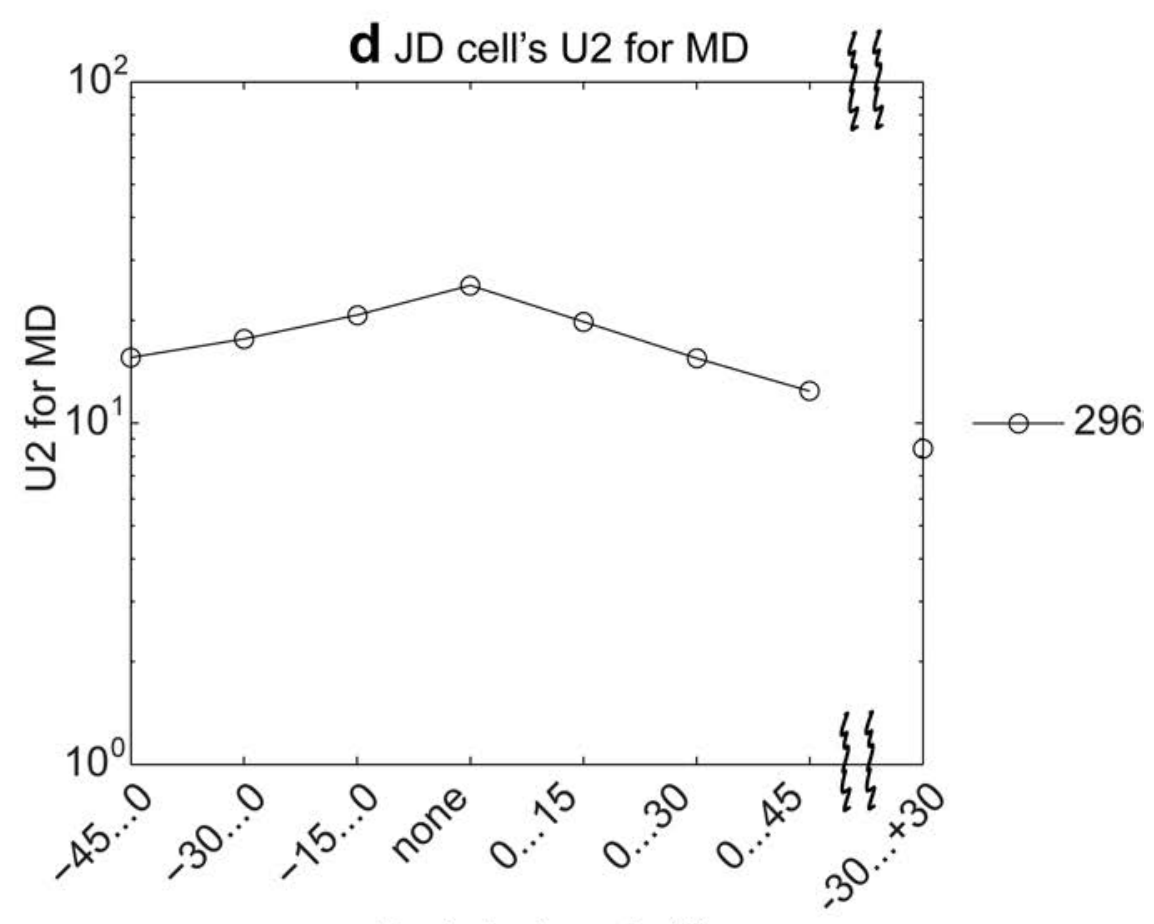

Excluded angle $\left({ }^{\circ}\right)$ 
Figure 5

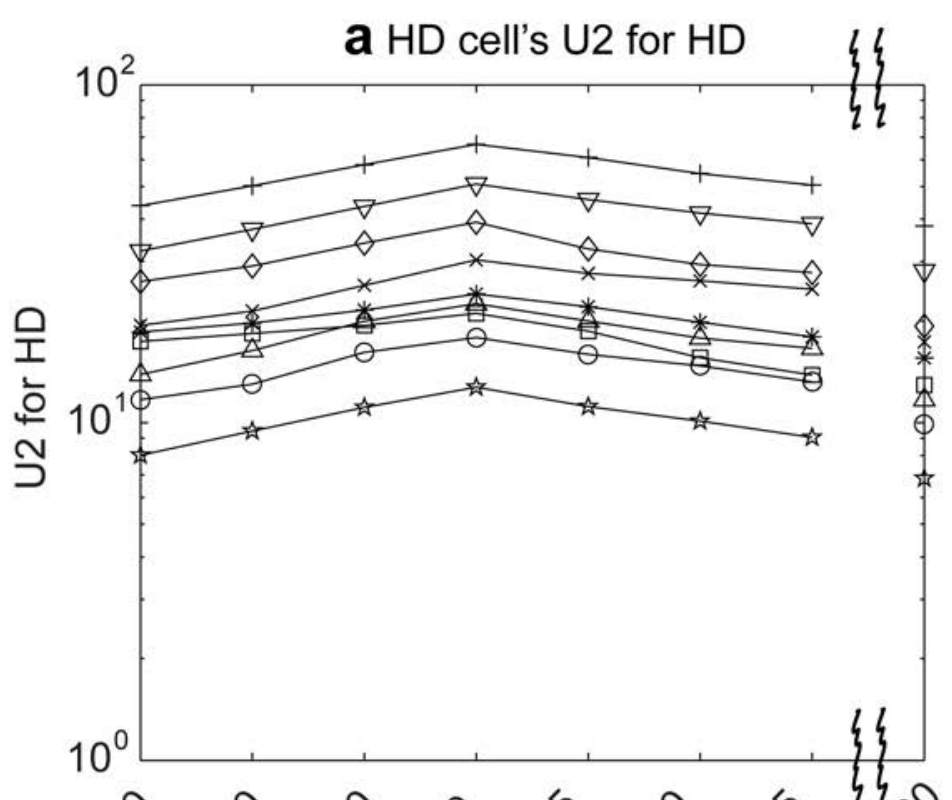

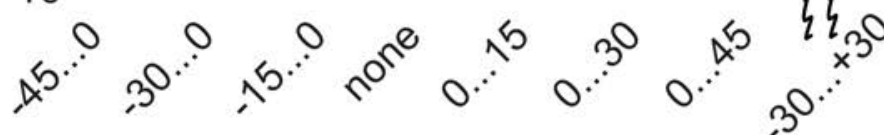

Excluded angle $\left({ }^{\circ}\right)$

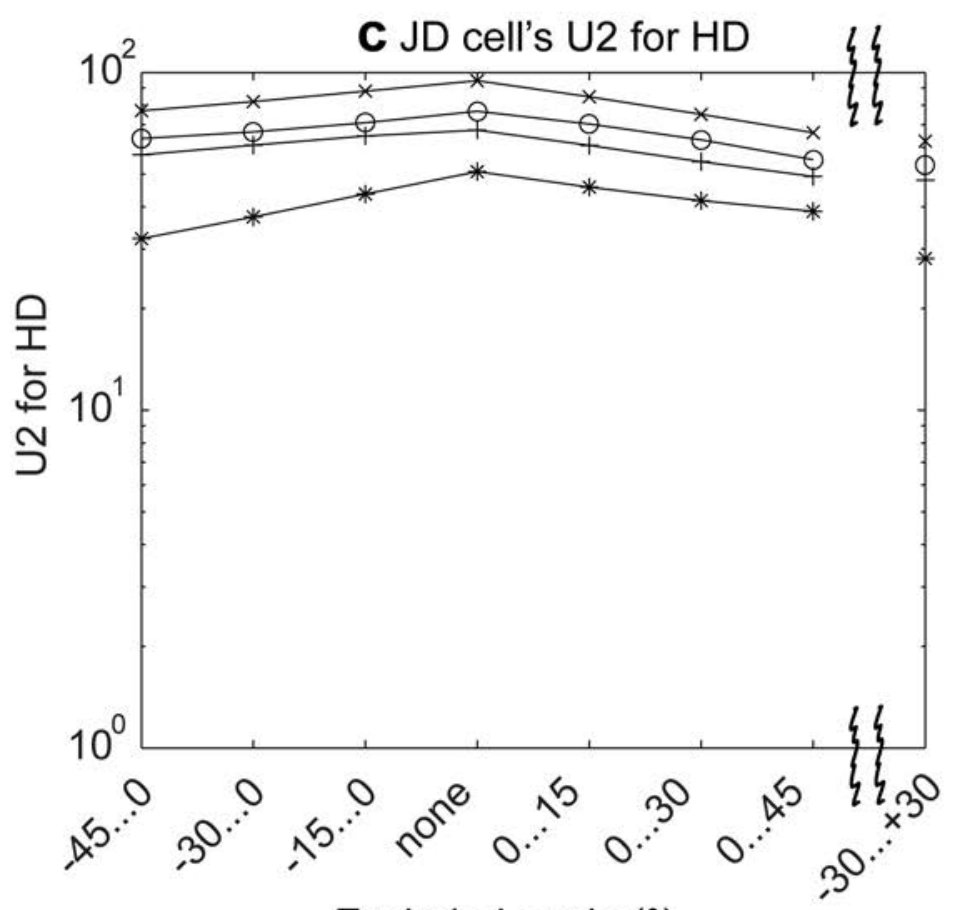

Excluded angle $\left({ }^{\circ}\right)$

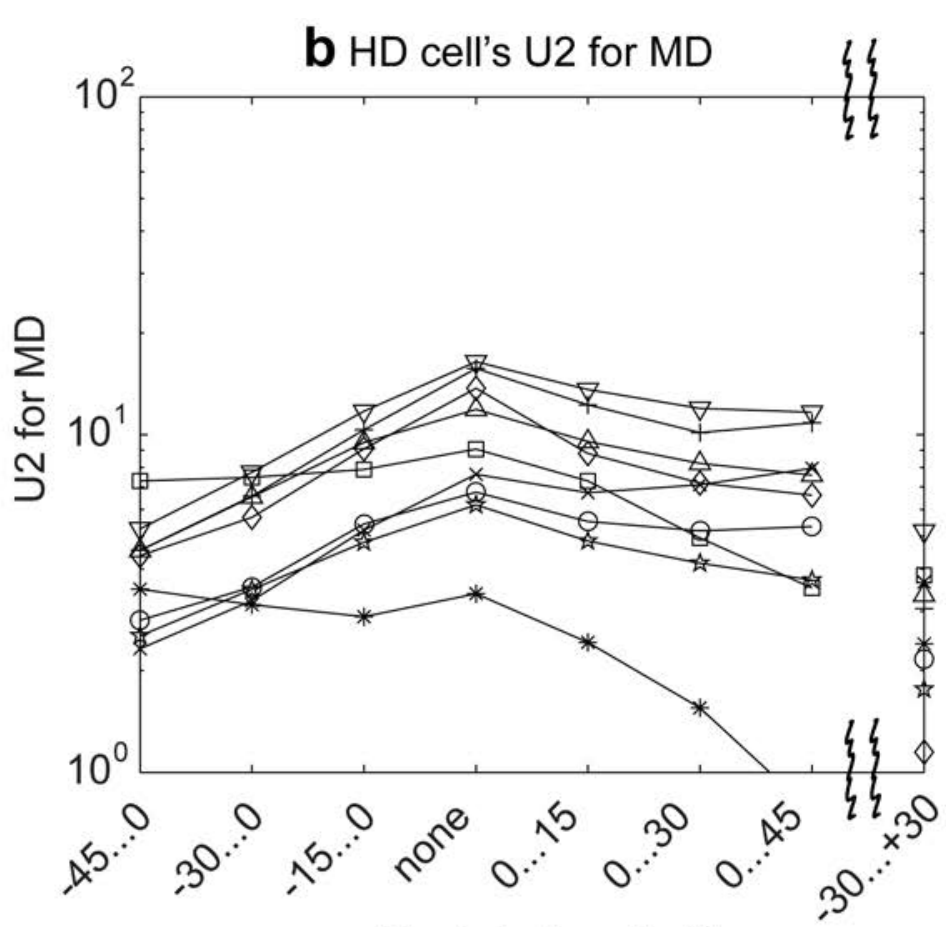

Excluded angle $\left({ }^{\circ}\right)$

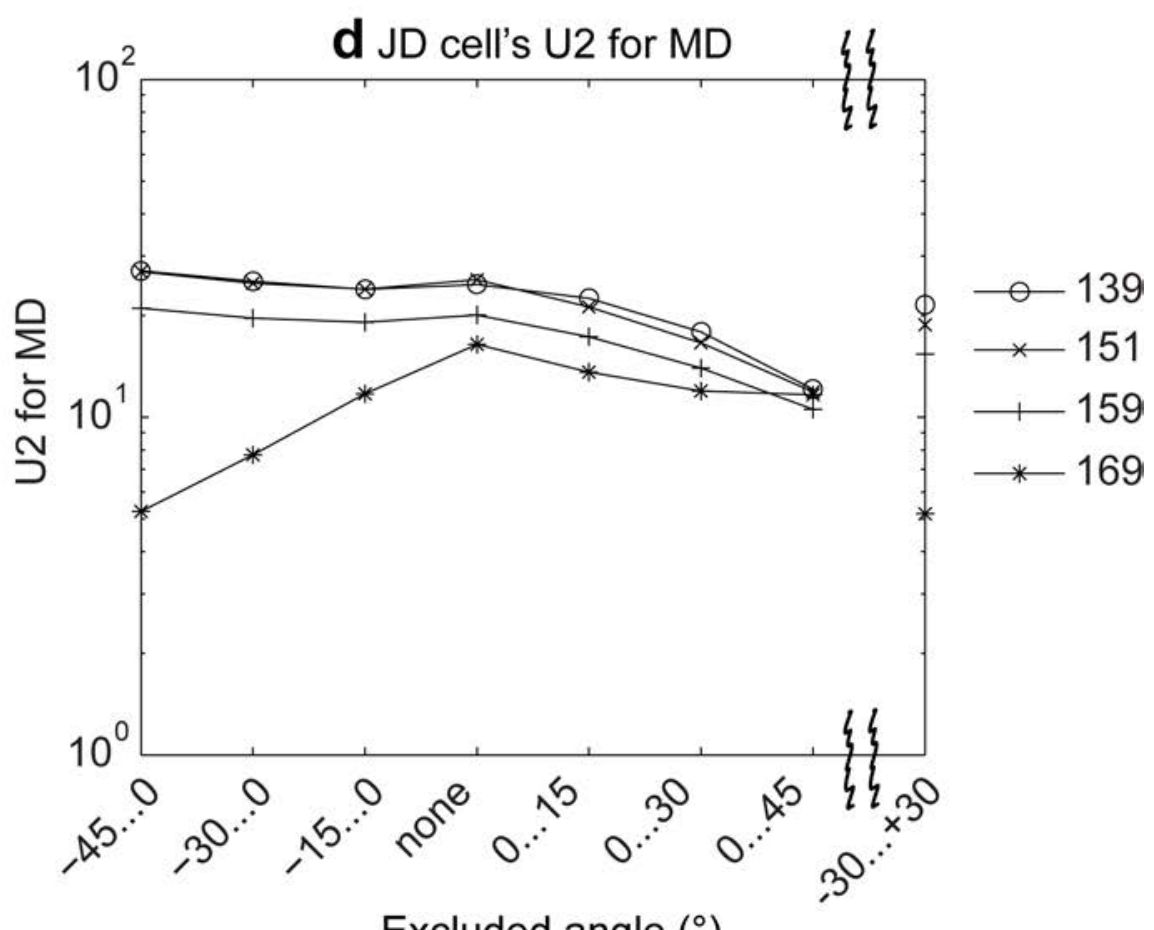

Excluded angle $\left({ }^{\circ}\right)$

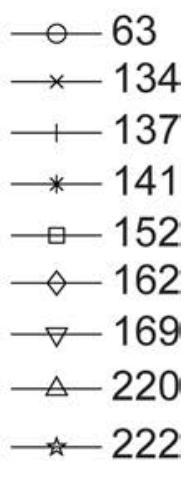

$\times-151$

$+159$

69
$-139$ 
Figure 6

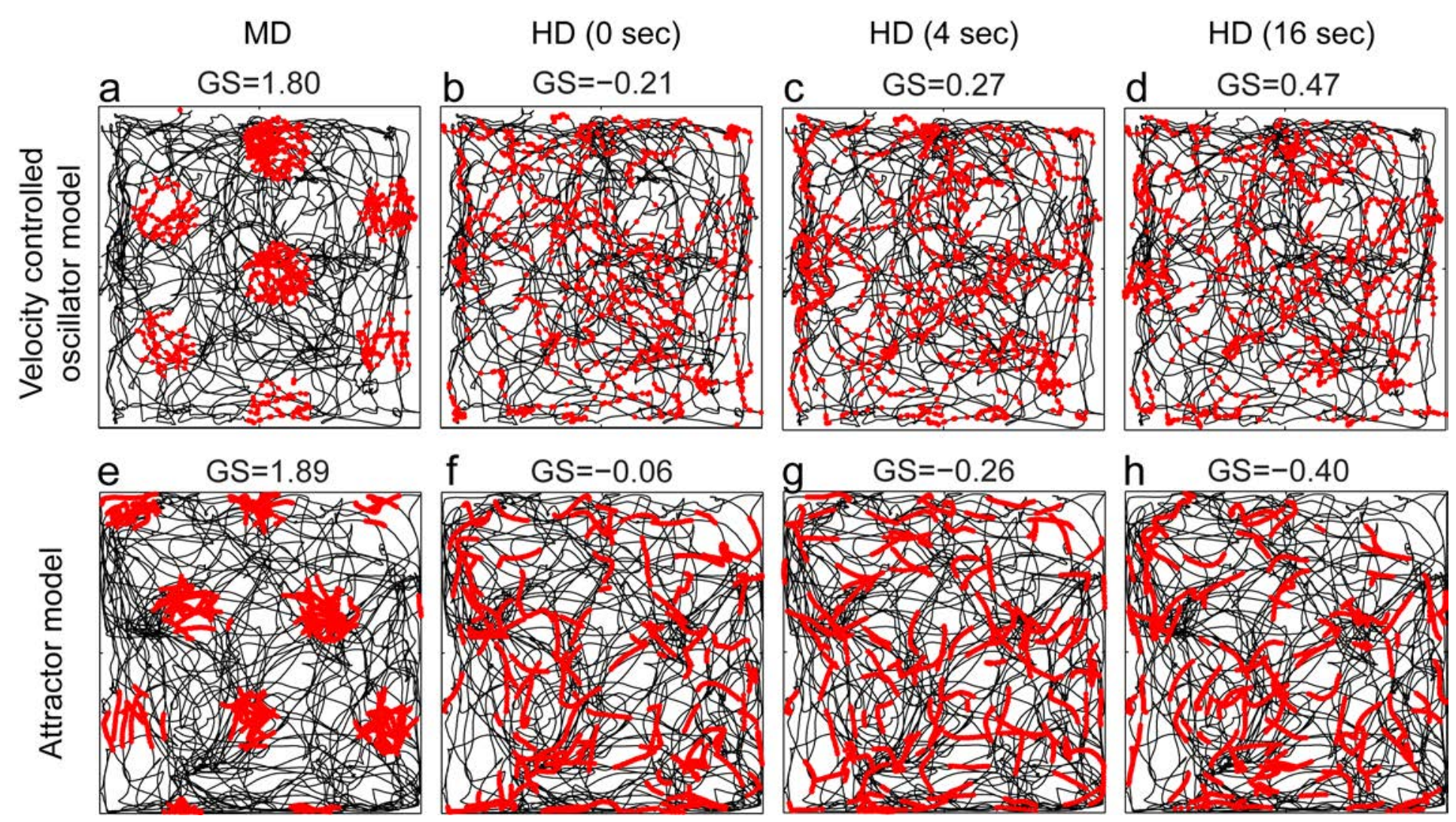

